\title{
APPLICATION OF PARAMETRIC MODELING TECHNOLOGY IN SINGLE SCREW EXTRUDER
}

\author{
Zhang Jingzhi' ${ }^{1}$, Ning Tingzhou ${ }^{1 *}$, Gou Yanan ${ }^{1 *}$ \\ ${ }^{1}$ College of Mechanical and Electrical Engineering, Zaozhuang University, Zaozhuang 277160, Shandong, China \\ Emails: ning-tingzhou@163.com, hyynan@163.com
}

\begin{abstract}
In order to improve the modelling efficiency of the key part for single screw extruder, the parametric modelling method of the key part for single screw extruder was studied based on SolidWorks and Visual Basic. The results shows that the SolidWorks can be mobilized to generate the 3D model of the screw by inputting the dimensions of each part in the parametric modelling window, which effectively improves the modelling efficiency. This study also provides reference for parametric modelling of other non-standard components.
\end{abstract}

Keywords: Parametric Modelling; Extruder; Screw; SolidWorks; Visual Basic.

\section{Introduction}

Single screw extruder is mainly used for extruding soft, hard polyvinyl chloride, polyethylene and other thermoplastic plastics. It can process many kinds of plastic products, such as film blowing, pipe extruding, plate pressing and ribbon drawing. It can also be used for melting granulation [1-3]. Screw is the heart of extruder and the key component of extruder. Its performance determines the productivity, plasticizing quality, dispersion of filler, melting temperature and power consumption of an extruder [4-6].

Some scholars have studied the parametric modelling technology of screw. For example, Zhang Linxiao developed the 3D parametric design software of the extrusion system for twin screw extruder by Visual $\mathrm{C}++6.0$ and Pro/Toolkit of Pro/Engineer [7]. Wang Ke studied the modelling program of using UG/Open GRIP language to create the 3D entity of the function section for cone twin screw, which could be directly driven by parameters [8]. Liu Qinglei studied the whole parametric process of finite element analysis for the screw of the biomass molding machine by using the solid modelling function in the pretreatment module of ANSYS and the secondary development languages APDL and UIDL [9]. Using the parametric design language provided by ANSYS, Kang Bing realized the parametric modelling of the cone screw with variable root diameter and pitch of the extruding bulking machine, and improved the modelling efficiency of the screw with complex curved surface
[10]. However, the parametric modelling method of screw using SolidWorks is less common.

Because of the complex structure of screw and the tedious modelling process, the parametric modelling method of screw is studied based on SolidWorks and Visual Basic. A set of fast modelling program of key part for single screw extruder is created to improve the modelling efficiency.

\section{Structure and Working Principle of Single Screw Extruder}

\subsection{Structure of single screw extruder}

As shown in figure 1, the structure of single screw extruder is mainly composed of transmission device, feeding device, heater, barrel, screw and other components.

\subsection{Working principle of single screw extruder}

Plastic material enters the extruder from feeding device and is conveyed forward by the screw. In the process of material moving forward, the material is melted by the heating of the barrel, shearing and compression are brought by the screw, thus realizing the change among the three states of glass, high elasticity and viscous flow. In the case of pressure, the material in the viscous flow state passes through a die with a certain shape, and then becomes a continuum with the same cross section and shape according to the die. Then the glass state is formed by cooling device, and the required parts are obtained. 


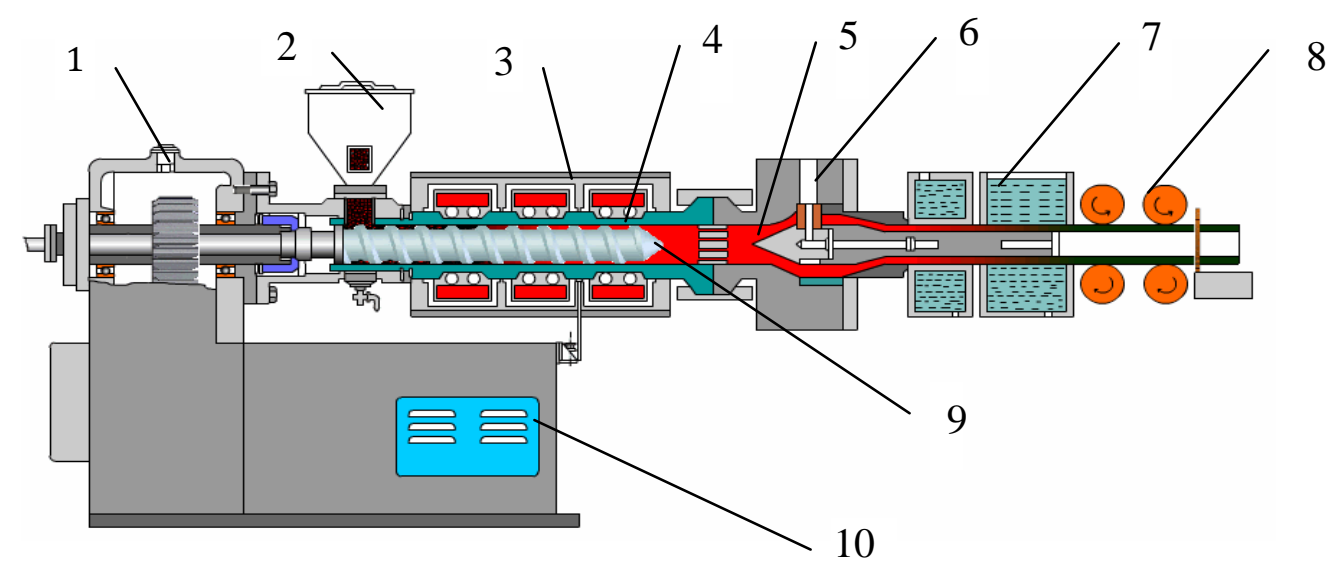

1. Transmission device; 2. Feeding device; 3. Heater; 4. Barrel; 5. Divider; 6. Handpiece; 7. Cooling device; 8. Tractive device; 9. Screw; 10. Electric cabinet

Figure 1. Structure of single screw extruder

\section{Key Parts of Single Screw Extruder}

As the material moves forward along the screw, it experiences changes in temperature, pressure and viscosity, which are different in the full length range of the screw. According to the changing characteristics of materials, the screw can be divided into feeding section, compression section and homogenization section [11].

\subsection{Feeding section}

The function of the feeding section is to send the material supplied by the hopper to the compression section. The plastic generally remains solid in the process of movement and partially melts due to heat. Depending on the type of plastic, the length of feeding section can start from the hopper to the end of $75 \%$ of the total length of the screw cup. Generally speaking, the extruded crystalline polymer is the longest, followed by the hard amorphous polymer, and the soft amorphous polymer is the shortest. Because the feeding section doesn't necessarily have compression effect, the volume of the screw groove can remain unchanged, while the size of the screw angle has a greater impact on the feed capacity of this section, which actually affects the productivity of the extruder. Generally, the productivity is highest when the spiral angle of powdery material is about $30^{\circ}$, the spiral angle of square block material should be about $15^{\circ}$, because the spherical material should be about $17^{\circ}$.

Main parameters of feeding section: Helix angle $\psi$ is generally from $17^{\circ}$ to $20^{\circ}$.The screw groove depth $\mathrm{H} 1$ is calculated by the geometric compression ratio of the screw $\varepsilon$ after the screw groove depth is determined in the homogenized section. The feeding section length L1 is determined by the empirical formula: for amorphous polymers, $\mathrm{L} 1=(10 \%-20 \%) \mathrm{L}$, for crystalline polymers, $\mathrm{L} 1=(60 \%-65 \%) \mathrm{L}$.

\subsection{Compression section}

The function of the compression section is to press the material, so that the material from the solid into the molten, and remove the air in the material. In order to adapt to the characteristics of volume reduction when the gas in the material is pushed back to the feeding section, the material is pressed and the material is melted, the screw in this section should have a greater shear and compression effect on the plastic. For this purpose, the volume of the screw groove is usually reduced gradually, and the degree of reduction is determined by the compressibility of the plastic. The compression ratio is not only related to the compression ratio of plastic, but also related to the shape of plastic. If the specific gravity of powder is small and the entrainment of air is large, the compression ratio needs to be to $4 \sim 5$, while the granular material is only $2.5 \sim 3$.

The length of compression section is mainly related to the melting point and other properties of plastics. The wider the melting temperature range, the longer the compression section, for example, PVC begin to melt above $150^{\circ} \mathrm{C}$, it's compression section is the longest, up to the full length of the screw, melting temperature range narrow polyethylene, compression section for the screw full length of $45 \sim 50 \%$. For most polymers with a narrow melting temperature range, the compression section is even only one pitch long, such as polyamide.

Main parameters of melting section: Compression ratio $\varepsilon$ : generally refers to the geometric compression ratio, which is the ratio of the volume of the first screw groove in the feeding section and the volume of the last screw groove in the homogenization section.

$\varepsilon=($ Ds-H1)H1/(Ds-H3) $\approx \mathrm{H} 1 / \mathrm{H} 3$

Where, $\mathrm{H} 1$ is the depth of the first screw groove in the feeding section, and H3 is the depth of the last screw groove in the homogenization section. 
The melting section length L2 is determined by the empirical formula: for amorphous polymers, $\mathrm{L} 2=55 \% \sim 65 \% \mathrm{~L}$, for crystalline polymers, $\mathrm{L} 2=(1 \sim$ 4)Ds.

\subsection{Homogenization section}

The function of the homogenization section is to feed the molten material into the head at constant quantitatively and pressure so that it can be formed in the die. The screw groove volume of homogenized section is as constant as that of feeding section. The screw head is usually conical or semicircular in design in order to avoid material decomposition caused by the material being trapped in the dead corner of the end of the screw head. Some of the homogenization sections are completely smoothed rod bodies called torpedo heads, but some are notched or milled into patterns. The torpedo head has the function of stirring and controlling the material, eliminating the pulsation phenomenon during the flow, reducing the thickness of the material layer, improving the heating condition, and further improving the plasticizing efficiency of the screw. This section may be $20-25 \%$ of the total screw length.

Important parameters of the homogenization section: The depth of screw groove $\mathrm{H} 3$ is determined by empirical formula, $\mathrm{H} 3=(0.02 \sim 0.06) \mathrm{Ds}$. The length L3 is determined by the formula, L3= $(20 \%$ $25 \%$ ) L.

\section{Selection of Parametric Softwares and Parametric Modelling Method 4.1 Modelling software}

SolidWorks is a powerful 3D modelling software, and it has a parametric design Interface (API) which provides researchers with secondary development [12-14]. By invoking the API, the repetitive, monotonous, or rule-specific operations of SolidWorks can be described in some programming language. These work can be done by the computer through simple human-computer interaction. Programming languages that support OLE and COM (such as Visual Basic, $\mathrm{C}++$, etc.) can be developed as the second development tool for SolidWorks.

\subsection{Programming language}

Visual Basic 6.0 is a visual, object-oriented, advanced computer programming language [15].
There is no need to write a lot of codes when use Visual Basic 6.0 to develop SolidWorks, only place object controls provided by the system on the interface according to the requirements of the specific design, and complete the corresponding object attribute setting and program writing. In this way, the design efficiency will be significantly improved.

\subsection{Parametric modelling method}

Parametric modelling method is shown in figure 2, mainly including: setup of entity model in SolidWorks, obtaining and modification of the variables in the equation, writing of the program in Visual Basic 6.0, establishment of user parametric interface, finally a set of parametric modelling program of the key part for single screw extruder [16].

Users can enter the interactive system interface through the parametric modelling program of the key part for single screw extruder. In accordance with the requirements, corresponding data of parameters can be input into the interactive system interface, finally, the 3D model of the part would be rebuilt in the screen.

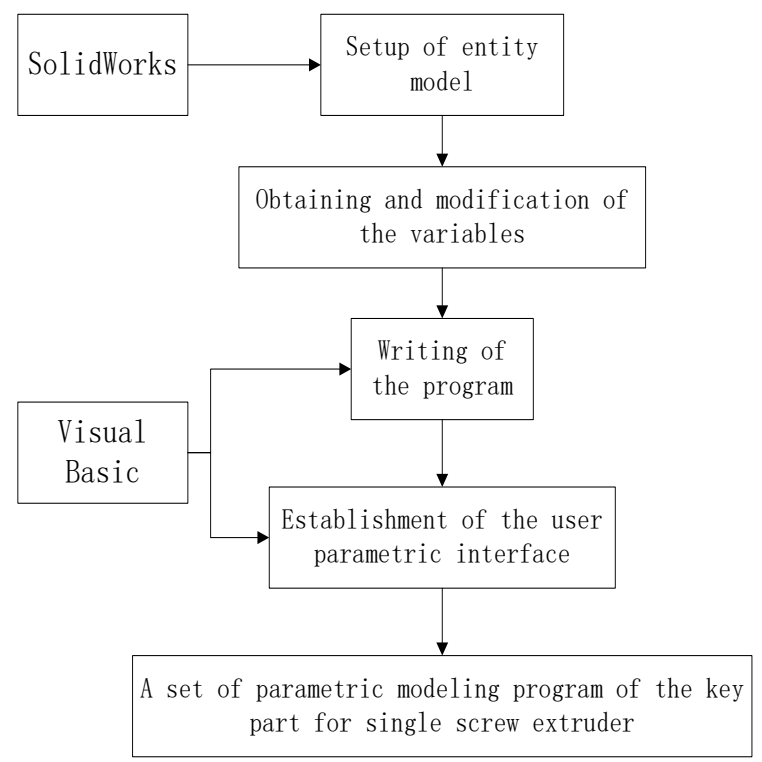

Figure 2. Parametric modelling method

\section{Parametric Modelling of Screw 5.1 Modelling}

In the 3D modelling software SolidWorks, the screw can be modelled by the extruded (including cutextruded), revolved, sweeping, chamfering and other operations. The modelling window of screw is shown in figure 3 . 


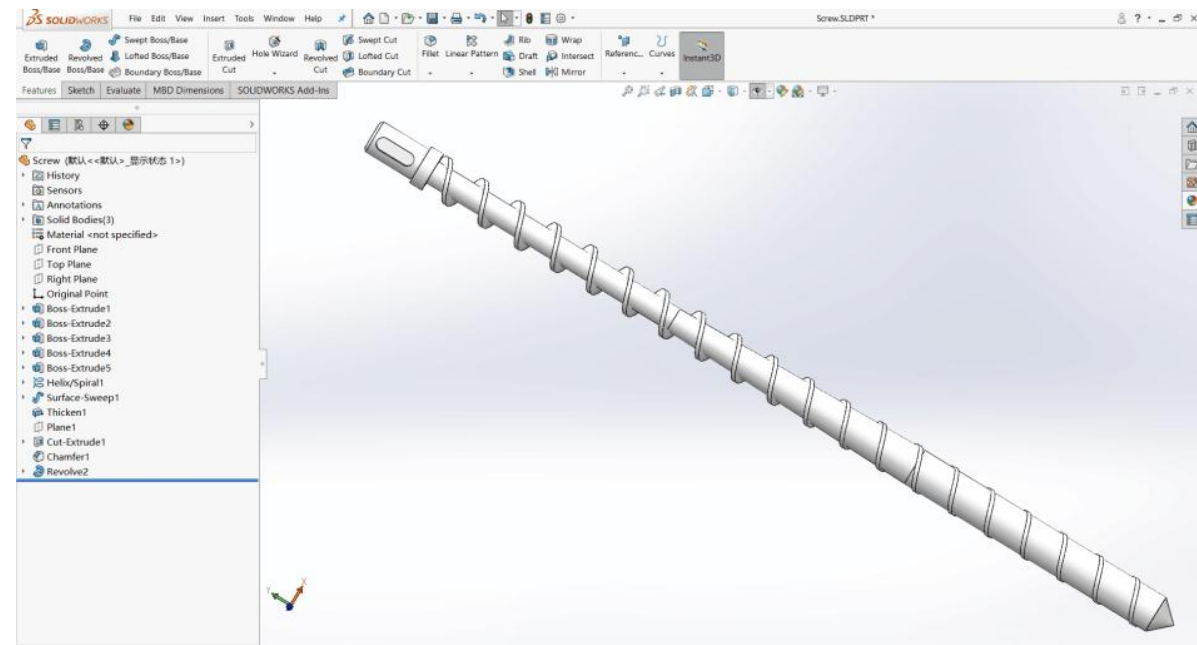

Figure 3. The modeling window of screw

\subsection{Programming}

After completing the entity modelling, click 'Equations' in 'Tools' to enter the equation interface

so as to view the attributes of all dimensions, as shown in figure 4, including all parameters that must be involved in the parameterized modelling.

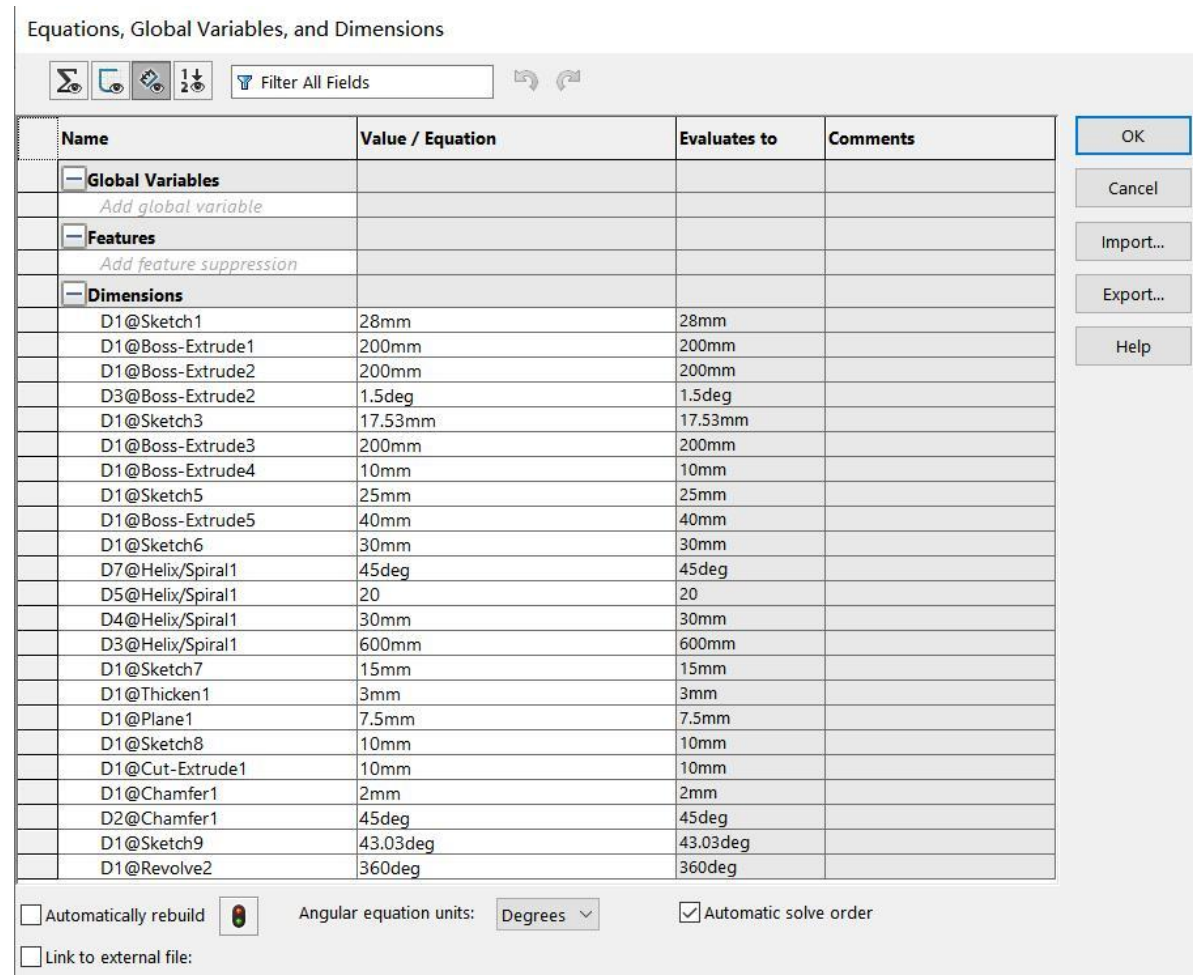

Figure 4. Equations of screw

According to the above equation results, Visual Basic programming is adopted to obtain the parametric codes of screw, the parametric codes are as follows.

Private Sub Command1_Click()

Dim swApp As Object ' Define variables for SolidWorks objects
Dim Part As Object ' Define variables for ModelDoc objects

Dim strfilename As String ' Define path variables Dim longstatus As Long ' Define the value of the state variable

Dim L1 As Double ' Define value type Dim L2 As Double ' Define value type Dim L3 As Double ' Define value type Dim Db As Double ' Define value type 
Dim Ds As Double ' Define value type

Dim t As Double ' Define value type

Dim $\theta$ As Double ' Define value type

Dim a As Double ' Define value type

Dim $\psi$ As Double ' Define value type

If Dir(strfilename) = "" Then

MsgBox ("File does not exist, please check:" \& vbCrLf \& strfilename) ' Determine whether the parameterized file exists

Set swApp = CreateObject(SldWorks.Application) ' Activate SolidWorks

Exit Sub

End If

strfilename

"C: \Users $\backslash 10194 \backslash$ Desktop \Screw.SLDPRT" ' Specify the path of the parameterized $3 \mathrm{~d}$ model file

Set swApp = CreateObject("SldWorks.Application") ' Activate SolidWorks

swApp.Visible $=$ True ' Software visualization

Set Part = swApp.OpenDoc4(strfilename, 1, 0, "', longstatus) ' Establish hold mode

Set Part = swApp.ActivateDoc("Screw.SLDPRT") '

Define the opening of part file name

L1 = Text2.Text ' Input parameter value

L2 = Text3.Text ' Input parameter value

L3 = Text4.Text ' Input parameter value

$\mathrm{a}=$ Text5.Text ' Input parameter value

Ds = Text8.Text ' Input parameter value

$\mathrm{Db}=$ Text9.Text ' Input parameter value

$\mathrm{t}=$ Text12.Text ' Input parameter value

$\theta=$ Text6.Text ' Input parameter value

Part.Parameter("D1@Sketch1@Screw.SLDPRT").syst emValue $=\mathrm{Db} / 1000{ }^{\prime}$ Define outer diameter of homogenization section

Part.Parameter("D1@Boss-

Extrude1@Screw.SLDPRT").systemValue = L3 /

1000 ' Define the length of homogenization section
Part.Parameter("D1@Boss-

Extrude1@Screw.SLDPRT").systemValue = L2 /

1000 ' Define the length of compression section

Part.Parameter("D3@Boss-

Extrude2@Screw.SLDPRT").systemValue = $\theta *$ 3.141592653 / 180 ' Define the taper of compression section

Part.Parameter("D1@Sketch3@Screw.SLDPRT").syst emValue $=(\mathrm{Db}-2 * \mathrm{~L} 2 * \theta * 3.141592653 / 180) /$ 1000 ' Define outer diameter of feeding section

Part.Parameter("D1@Boss-

Extrude3@Screw.SLDPRT").systemValue = L1 / 1000 ' Define the length of feeding section

Part.Parameter("D1@Sketch6@Screw.SLDPRT").syst emValue $=$ Ds $/ 1000$ ' Define the outside diameter of the helix

Part.Parameter("D5@Helix/Spiral1@Screw.SLDPRT "). systemValue $=(\mathrm{L} 1+\mathrm{L} 2+\mathrm{L} 3) / \mathrm{t}$ ' Define the number of the helix

Part.Parameter("D4@Helix/Spiral1@Screw.SLDPRT ").systemValue $=\mathrm{t} / 1000$ ' Define the pitch of the helix

Part.Parameter("D3@Helix/Spiral1@Screw.SLDPRT "). systemValue $=(\mathrm{L} 1+\mathrm{L} 2+\mathrm{L} 3) / 1000$ ' Define the length of the helix

Part.Parameter("D1@Thicken1@Screw.SLDPRT").sy stemValue $=\mathrm{a} / 1000$ ' Define the thickness of the helix

Part.EditRebuild ' Update the file

Set Part $=$ Nothing ' Release Part variable pointer

Set swApp $=$ Nothing ' Release swApp variable pointer

End Sub

Private Sub Command2_Click()

End ' Exit the program

End Sub

The parametric modelling window of screw is shown in figure 5.

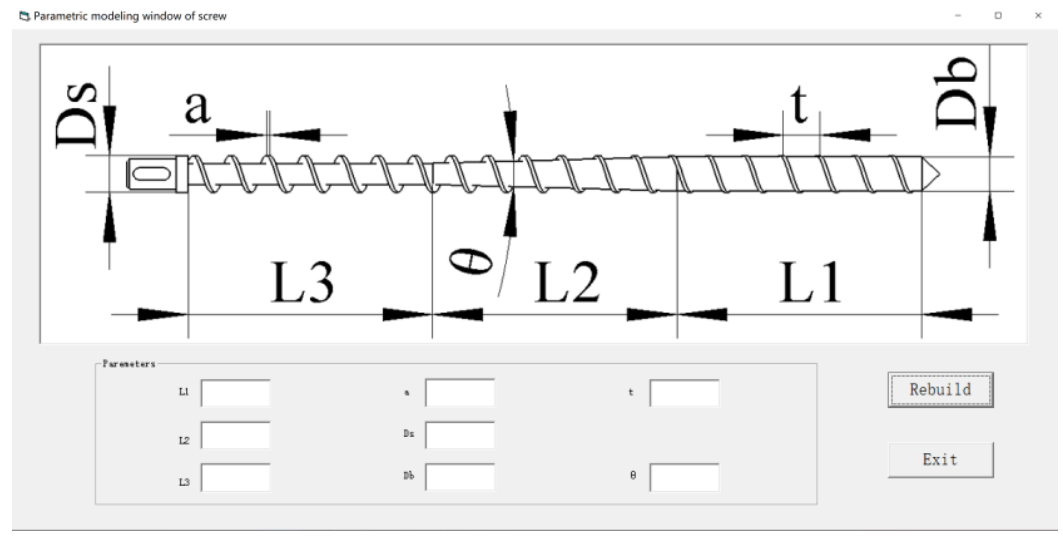

Figure 5. Parametric modeling window of screw

\subsection{An exemple of parametric modelling of screw}

Enter the required size values in the parametric modelling window of screw shown in figure 5 , then click 'Rebuild' to generate the required screw, as shown in figure 6. 


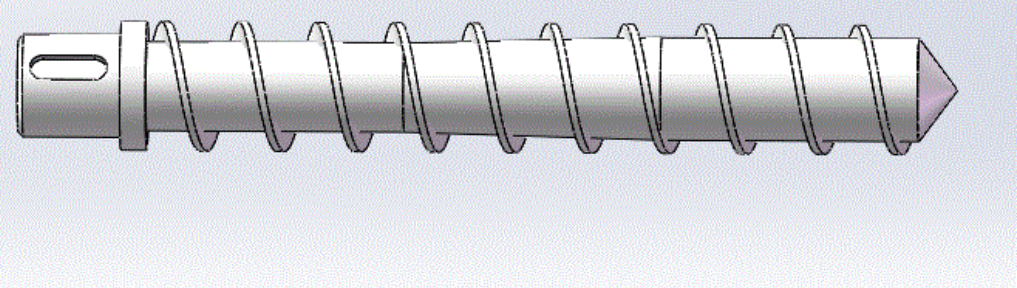

Figure 6. New model of screw

\section{Conclusion}

Parametric modelling of key part for single screw extruder is realized based on SolidWorks and Visual Basic 6.0. Through the developed program, the 3D model datum of screw can be modified directly, and the new model of screw can be customized.

\section{Acknowledgement}

This work was supported by Zaozhuang Science and Technology Plan Project (2019GX10) and Zaozhuang University Doctoral Research Startup Fund Project (2018BS030).

\section{References}

[1] Wilczyński K J, Lewandowski A,Wilczyński K.(2016). Experimental study of melting of polymer blends in a starve fed single screw extruder. Polymer Engineering \& Science, 56(12), 1349-1356.

[2] Altınkaynak A, Gupta M, Spalding M A, et al. (2011). Melting in a single screw extruder: experiments and 3D finite element simulations. International Polymer Processing, 26(2), 182196.

[3] Singh S K, Muthukumarappan K. (2017). A viscosity model for soy white flakes-based aquafeed dough in a single screw extruder. Journal of Food Process Engineering, 40(2), e12357.

[4] Abeykoon C. (2016). Single screw extrusion control: A comprehensive review and directions for improvements. Control Engineering Practice, 51, 69-80.

[5] Chen K, Cui Y, Wang S, et al. (2020). Characterization of plasticizing process of single screw extruder with grooved melting zone. Journal of Polymer Research, 27(3), 1-11.

[6] Han Y, Jia F, Zeng Y, Jiang L, et al. (2017). DEM study of particle conveying in a feed screw section of vertical rice mill. Powder technology, 311, 213-225.
[7] Zhang Linxiao,Zhou Yongling.(2010).3D parametric design of twin-screw extruder extrusion system based on Pro/E.Technological Development of Enterprise, 29(1), 28-31.

[8] Wang Ke,Han Licen,Sun Xingwei.(2010). Research on Parametric Modeling of Conical Twin-screw Based on UG Software. Light Industry Machinery, 28(3), 38-40.

[9] Liu Qinglei,Dong Yuping,Chang Jiafu,et al.(2013).Parametric Modeling and Analysis of Screw Shaft of Biomass Feeder Based on Apdl. Journal of Agricultural Mechanization Research, 35(4), 218-221.

[10] Kang Bing,Zhang Zuli.(2007). The Parametric Modeling of Extruding Taper Screw Rod Based on APDL. Journal of Agricultural Mechanization Research, 4,56-58.

[11] Li Huanhuan, (2016). Structural optimization and performance analysis of single-screw for wheat flour.Wu Xi: Jiangnan University.

[12] LIU Min and HE Zhi-min. (2013).Parametric Design Research on the Parts of Stepped Shaft Based on Solidworks. Computer Programming Skills \& Maintenance, 6,62-65.

[13] Li Yan, Long Huicai, Wang Yilin, et al. (2014). Parametric design on working parts of fine blanking progressive dies based on SolidWorks. Forging \& Stamping Technology, 39(10), 140145.

[14] Chen Chen, Li Hua, Zeng Yi.(2017).Application of SolidWorks Secondary Development in the Parametric Modeling of Flange for Pressure Vessel. Chemical Equipment Technology, 38(2), 33-35+43.

[15] ZHANG Haitao and XU Guangquan. The development and application of aquifer hydrogeolgical parameter calculation software based on Visual Basic 6.0. Coal Geology \& Exploration, 46(2), 105-110.

[16] HAN Ze-Cuang, SUN Jun-Xiang, HAO Rui-Qin. (2017). The Second Development of SolidWorks Parametric Design Based on VB. Development \& Innovation of Machinery \& Electrical Products,30(5),79-81. 